\title{
Criterios de laboratorio clínico y su utilidad como predictores del diagnóstico de síndrome cardiopulmonar por hantavirus
}

\author{
Marina Opazo', Diego Guerrero', Ximena Collao², Cindy Peña ${ }^{2}$ y Humberto Villalobos ${ }^{3}$
}

\section{Clinical laboratory criteria and its utility as predictive of diagnosis of the cardiopulmonary syndrome by hantavirus}

Background. The hantavirus infection is an emerging zoonotic disease, endemic in Chile, generating the hantavirus cardiopulmonary syndrome (HCPS), characterized by cardiopulmonary dysfunction with rapidly progressive respiratory failure and high lethality. For an early clinical orientation of HCPS, due to its non-specificity in symptoms and to help the differential diagnosis, some laboratory parameter that may be useful have been studied. Aim: To identify laboratory criteria as predictive factors of HCPS in patients with suspected hantavirus infection. Methodology: Retrospective cohort study of 71 patients admitted to the Hospital Guillermo Grant Benavente Emergency. We determined discriminative capacity of laboratory's parameters at the time of admission: platelets recount, hematocrit, inmunoblasts, activated partial thromboplastin time (aPTT) and aspartate aminotransferase (AST/GOT). Results: Were found significant differences in all parameters studied between confirmed patients (22) with respect to unconfirmed (49). Hematocrit, inmunoblasts, AST/GOT and aPTT had a OR $>1$ and platelets count had a OR $<1$. The best combination for predict HCPS was hematocrit, platelets count and AST/GOT with $90,01 \%$ sensibility and $81,63 \%$ specificity. Conclusion: The five parameters studied are good predictors of HCS in suspicious patients and they would may be useful in low complexity hospitals for quick transfer a center with critical care units.

Keywords: Hantavirus; diagnosis; hantavirus cardiopulmonary syndrome; laboratory parameters.

Palabras clave: Hantavirus, diagnóstico, síndrome cardiopulmonar por hantavirus, valores de laboratorio.

\section{Introducción}

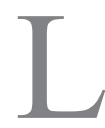

os hantavirus son virus de ARN de hebra simple, circular y de polaridad negativa, que pertenecen a la familia Bunyaviridae e infectan a muchas especies de roedores, musarañas, topos y murciélagos. La infección en estos hospederos es generalmente asintomática y algunos roedores transmiten la enfermedad a los humanos pudiendo causar dos patologías: fiebre hemorrágica con síndrome renal (HFRS) y síndrome cardiopulmonar por hantavirus $(\mathrm{SCPH})^{1}$.

El agente etiológico del SCPH en nuestro país es el virus Andes (ANDV). Su reservorio principal es el "ratón de cola larga" (Oligoryzomys longicaudatus) el que se distribuye desde la Región de Atacama hasta la Región de Magallanes. El principal mecanismo de transmisión de ANDV a los humanos es a través de la inhalación de aerosoles conteniendo partículas virales provenientes de la orina o saliva de roedores infectados, o bien, por el contacto directo con sus excretas ${ }^{2}$.

El SCPH evoluciona en tres fases; prodrómica, cardiopulmonar y convalecencia. El período de incubación fluctúa entre 1 y 3 semanas, con un rango de 7 a 45 días. La fase prodrómica se extiende entre 1 a 6 días con síntomas inespecíficos, como fiebre, mialgias, cefalea, náuseas, vómitos, dolor abdominal, diarrea y artralgias. La fase cardiopulmonar se caracteriza por aparición brusca de tos, dificultad respiratoria (disnea) e inestabilidad hemodinámica (hipotensión arterial) secundarios al compromiso de la permeabilidad capilar que afecta predominantemente al pulmón ${ }^{3}$. Los sujetos que sobreviven la fase de SCPH inician la fase de convalecencia, durante la cual se produce una recuperación completa de la función pulmonar y hemodinámica ${ }^{3}$. El tratamiento actual del SCPH se basa en la terapia de soporte circulatoria y respiratoria en Unidades de Paciente Crítico y puede requerir uso de circulación extracorpórea (ECMO) para evitar la muerte ${ }^{3}$.

En la fase prodrómica, la trombocitopenia es la anormalidad más consistente como sospecha de serología positiva para hantavirus ${ }^{4,5}$. En la fase pulmonar además se evidencian: mielocitos, hemoconcentración, falta de granulación toxica significativa en neutrófilos y más de $10 \%$ de linfocitos con características morfológicas de inmunoblastos, todos ellos con sensibilidad de $96 \%$ y una especificidad de $99 \%$ para $\mathrm{SCPH}^{6}$. Se ha demostrado también que, en pacientes confirmados, la urea, creatinina ácido úrico, lactato deshidrogenasa
'Laboratorio Clínico del Hospital Guillermo Grant Benavente de Concepción. Concepción, Chile. 2Laboratorio de Virología, Escuela de Medicina, Universidad de Valparaíso. Valparaíso, Chile. ${ }^{3}$ Oficina de Investigación, Escuela de Medicina, Universidad de Valparaíso. Valparaíso, Chile.

Fuente de financiamiento: Laboratorio, Hospital de Concepción.

Sin conflictos de interés.

Recibido: 20 de marzo de 2018 Aceptado: 21 de febrero de 2019

Correspondencia a: Marina Opazo Riveros mopazo@ssconcepcion.cl 
(LDH), aspartato transaminasa (AST/GOT), fosfatasa alcalina (ALP) y proteína $\mathrm{C}$ reactiva (PCR), son significativamente elevados y el recuento de plaquetas significativamente bajo ${ }^{7}$.

En Chile, es insuficiente el conocimiento de los hallazgos de laboratorio que ayuden a predecir el SCPH, especialmente en las fases iniciales de la enfermedad, donde los síntomas y signos de la infección son inespecíficos y pueden confundirse o corresponder a otras patologías, generando muchas veces un diagnóstico tardío y que el paciente consulte varias veces antes de sospechar de este agente etiológico ${ }^{7}$. Se debe considerar que la evolución catastrófica del SCPH suele ser extremadamente rápida y difícil de predecir. Si el paciente se encuentra en un centro hospitalario de baja complejidad o en una zona rural apartada, ante un diagnóstico precoz se recomendaría el traslado precoz a un centro que cuente con Unidad de Paciente Crítico (UPC).

La situación en Chile y la observación local fueron los principales motivos para realizar este estudio con el fin de identificar parámetros de laboratorio que puedan contribuir tempranamente al diagnóstico del SCPH y a la toma de decisiones oportunas de manejo clínico y/o de traslado del paciente a un centro especializado para mejorar la sobrevida, mientras se realicen los exámenes confirmatorios.

\section{Materiales y Métodos}

\section{Diseño del estudio}

Se realizó un estudio analítico de cohorte retrospectiva cuya población de estudio fue constituida por un universo de 97 pacientes provenientes de áreas y hospitales rurales de la Región del Bío Bío de Chile, que fueron internados en el Hospital Guillermo Grant Benavente de Concepción (HGGB) con sospecha de SCPH, entre los meses de enero del año 2011 y diciembre de 2016. La confirmación de los casos sospechosos fue realizada tanto en el Instituto de Salud Pública (ISP) como en el laboratorio del HGGB, mediante reacción de polimerasa en cadena en tiempo real RPC-TR desarrollado por el ISP y transferido al laboratorio del HGGB y/o ELISA comercial "Hantavirus Pool 2 America", de la empresa Euroimmun, previamente validado por el ISP.

En 24 pacientes $(24,7 \%)$ fue confirmada la enfermedad por hantavirus y en 73 pacientes $(75,3 \%)$ fue descartada dicha infección. Criterios: Se incluyeron en el estudio, todos los pacientes que a su ingreso al HGGB se les realizaron los siguientes exámenes: Recuento de plaquetas, hematocrito, presencia de inmunoblastos, TTPa y GOT. Cohorte: Corresponde a aquellos pacientes que cumplieron los criterios de inclusión. Variables en estudio: La variable depen- diente analizada fue la presencia de hantavirus. Las variables independientes (parámetros de laboratorio): Recuento de plaquetas expresado $\times 10^{3} / \mu \mathrm{L}$, porcentaje del hematocrito, porcentaje de inmunoblastos, TTPa expresado en segundos, GOT expresado en UI/L. Los profesionales del área de Hematología del laboratorio clínico están constantemente capacitándose en la lectura de frotis patológicos, demostración de lo anterior es la participación constante y satisfactoria del programa de evaluación de calidad (PEEC) del ISP.

\section{Comparaciones entre los grupos de pacientes}

Se compararon los resultados de laboratorio de los pacientes con presencia de enfermedad por hantavirus v/s los pacientes con ausencia de la misma. Se evaluó la homogeneidad de los grupos de pacientes de la cohorte seleccionada y la normalidad en la distribución de los resultados de las variables independientes del estudio, mediante métodos de Shapiro-Wilks y KolmogorovSmirnov. Se evaluó si existe asociación entre el sexo y la confirmación de enfermedad por hantavirus, mediante el test exacto de Fisher. Se compararon las variables entre los dos grupos de pacientes, mediante el test estadístico U de Mann-Whitney. Se determinó la capacidad discriminativa de los parámetros para $\mathrm{SCPH}$ según los criterios de Hosmer y Lemshow ${ }^{9}$, se identificaron puntos de corte utilizando Curvas ROC, con sus respectivas coordenadas y su IC95\%. Mediante análisis de regresión logística se obtuvieron los odds ratio (OR) para cada uno de los cinco parámetros estudiados para medir la magnitud del efecto en la predicción del SPCH. Finalmente, mediante análisis discriminante se obtuvo la combinación de estos parámetros más idónea para predecir precozmente SCPH. Todos los análisis estadísticos fueron realizados con el software STATA 14 IC, y se consideró significativo a valores de $\mathrm{p}<0,05$.

Este estudio contó con la aprobación del Comité ÉticoCientífico del Servicio de Salud de Concepción. Los datos analizados en este estudio se obtuvieron de los sistemas de registros manuales e informáticos del Laboratorio (LIS) y del sistema informático del hospital (HIS). Los datos fueron recolectados resguardando la confidencialidad de la identidad de los pacientes.

\section{Resultados}

De un total de 97 casos sospechosos de enfermedad por hantavirus, sólo $71(73 \%)$ de ellos cumplieron los criterios de inclusión y fueron considerados como cohorte en este estudio. De ellos, 54 fueron de sexo masculino (19 confirmados como positivos y 35 como negativos), y 17 fueron de sexo femenino (tres confirmadas como positivos y 14 como negativos). No se encontró una asociación 
estadísticamente significativa entre el sexo y el estado de infección $(\mathrm{p}=0,235)$.

Debido que las variables estudiadas poseían una distribución no normal (resultados no mostrados), se realizó test U de Mann-Whitney para evaluar la diferencia entre las medianas. Los resultados se encuentran resumidos en la Tabla 1 y en la Figura 1 (A-E), donde se observa que hematocrito, inmunoblastos, GOT y TTPa se encuentran aumentados en el grupo de los positivos en comparación a los negativos; en cambio, las plaquetas se encuentran disminuidas en el primer grupo en comparación con el segundo.

\begin{tabular}{|c|c|c|c|c|c|}
\hline \multirow[t]{2}{*}{ Parámetro } & \multicolumn{2}{|c|}{ Positivos } & \multicolumn{2}{|c|}{ Negativos } & \multirow[t]{2}{*}{ Valor $p$} \\
\hline & Mediana & $\mathbf{R I}$ & Mediana & $\mathbf{R I}$ & \\
\hline Hematocrito (\%) & 48,3 & $41,7-53,0$ & 41,1 & $37,5-45,0$ & 0,0030 \\
\hline Plaquetas $\left(10^{3} / \mathrm{mm}^{3}\right)$ & 46 & $37-85$ & 190 & $123-238$ & $<0,0001$ \\
\hline Inmunoblastos (\%) & 17,5 & $0,0-37,5$ & 3,8 & $0,0-4,0$ & 0,0002 \\
\hline TTPa (segundos) & 46,4 & $36,5-70,5$ & 32,1 & $28,7-37,7$ & $<0,0001$ \\
\hline GOT (UI/mL) & 53,6 & $39,2-72,8$ & 31,0 & $22,0-56,0$ & 0,0002 \\
\hline
\end{tabular}

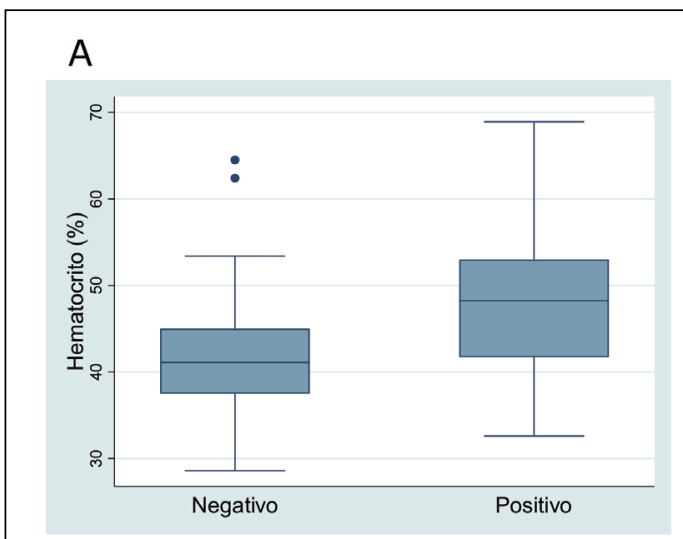

C

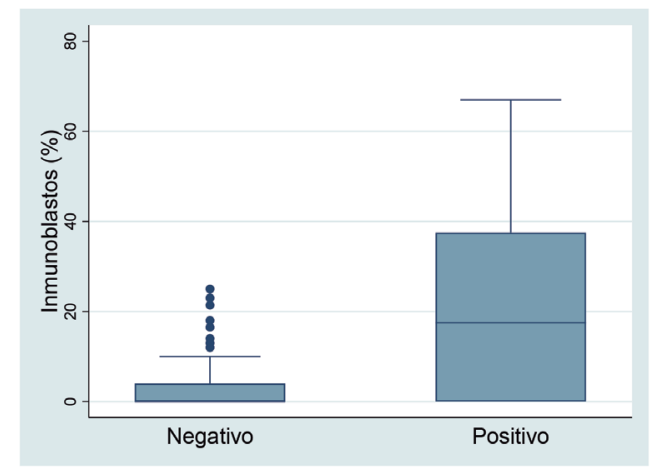

$\mathrm{E}$

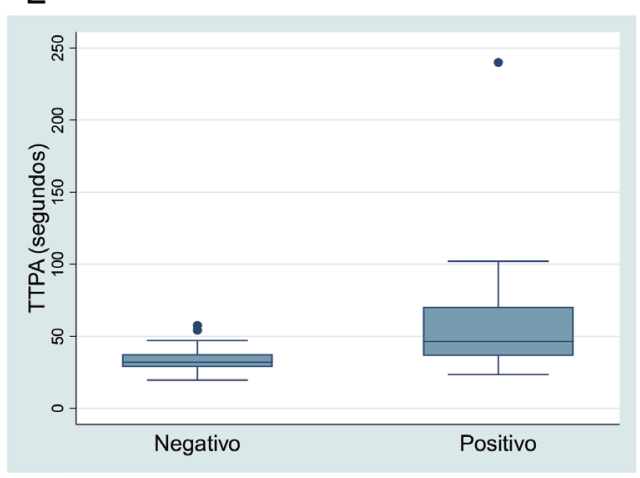

\section{B}

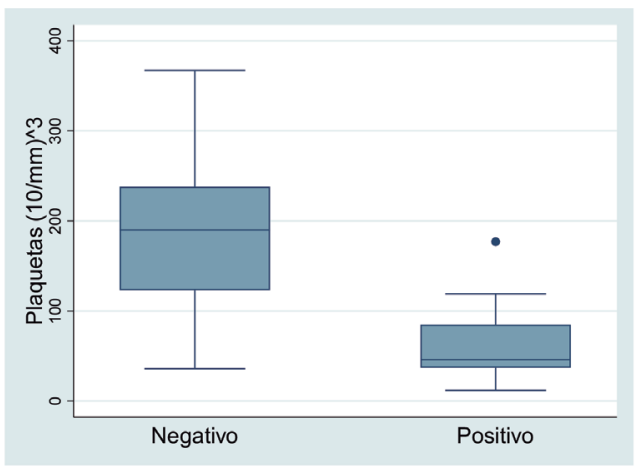

D

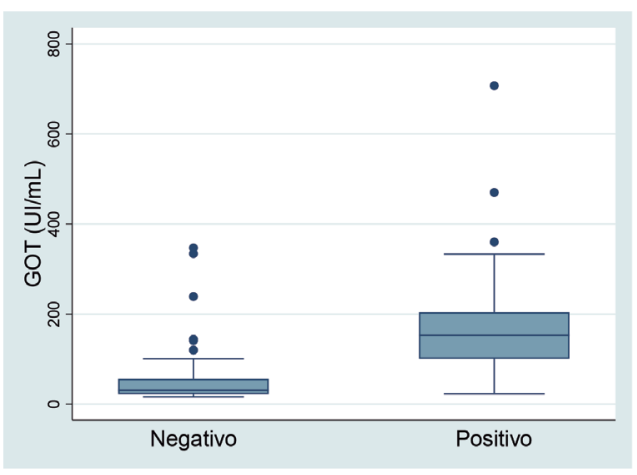

Figura 1. Box-plot de las variables estudiadas respecto al estado final de infección por hantavirus. (A) Hematocrito. (B) Plaquetas. (C) Inmunoblastos. (D) GOT. (E) TTPa. 
El área bajo la curva (AUC) de las curvas ROC con sus respectivos intervalos de confianza, se encuentran resumidas en la Tabla 2. En ella se observa, que para los cinco parámetros el AUC fue mayor a 0,5 y el poder

Tabla 2. Área bajo la curva de las respectivas curvas ROC con sus intervalos de confianza al $95 \%$

\begin{tabular}{lccc}
\hline Parámetro & AUC & Intervalo de confianza al 95\% & Discriminación* \\
Hematocrito $(\%)$ & 0,7217 & $0,57449-0,86893$ & Aceptable \\
Plaquetas $\left(10^{3} / \mathrm{mm}^{3}\right)$ & 0,9054 & $0,83596-0,97480$ & Excelente \\
Inmunoblastos $(\%)$ & 0,7542 & $0,62159-0,88676$ & Aceptable \\
TTPa (segundos) & 0,8066 & $0,68872-0,92445$ & Muy buena \\
GOT (Ul/mL) & 0,8595 & $0,76465-0,95427$ & Muy buena \\
\hline
\end{tabular}

AUC: Área bajo la curva. *En base a los criterios propuestos por Hosmer y Lemeshow ${ }^{8}$.

Tabla 3. Puntos de corte con su respectivo par sensibilidad/especificidad

\begin{tabular}{llccc}
\hline Parámetro & $\mathbf{n}$ & Punto de corte & $\begin{array}{c}\text { Sensibilidad } \\
\%\end{array}$ & $\begin{array}{c}\text { Especificidad } \\
\%\end{array}$ \\
\hline Hematocrito (\%) & 71 & $>47,3$ & 63,6 & 89,8 \\
\hline Plaquetas $\left(10^{3} / \mathrm{mm}^{3}\right)$ & 71 & $<119$ & 95,4 & 75,5 \\
Inmunoblastos (\%) & 71 & $>15,0$ & 59,0 & 89,8 \\
TTPa (segundos) & 71 & $>41,6$ & 77,2 & 73,4 \\
GOT (UI/mL) & 71 & $>69,0$ & 86,3 & 79,5 \\
\hline
\end{tabular}

Tabla 4. Odds ratio y coeficientes de regresión logística de los parámetros clínicos estudiados

\begin{tabular}{lccc}
\hline Parámetro & $\begin{array}{c}\text { Coeficiente de } \\
\text { regresión }\end{array}$ & OR & IC (95\%) \\
\hline Hematocrito $(\%)$ & 0,098 & 1,10 & {$[1,028-1,184]$} \\
Plaquetas $\left(10^{3} / \mathrm{mm}^{3}\right)$ & $-0,030$ & 0,97 & {$[0,956-0,985]$} \\
Inmunoblastos $(\%)$ & 0,097 & 1,10 & {$[1,044-1,163]$} \\
TTPa (segundos) & 0,092 & 1,10 & {$[1,041-1,153]$} \\
GOT $(\mathrm{Ul} / \mathrm{mL})$ & 0,013 & 1,01 & {$[1,005-1,020]$} \\
\hline
\end{tabular}

OR: odds ratio.

Tabla 5. Tabla de contingencia de análisis discriminante al combinar plaquetas, hematocrito y GOT

\begin{tabular}{lcc|}
\hline Clasificado & \multicolumn{2}{c}{ Perdadero estado } \\
\hline Positivo & $20(90,9 \%)$ & Negativo \\
Negativo & $2(9,1 \%)$ & $9(18,4 \%)$ \\
\hline
\end{tabular}

discriminativo fue por lo menos aceptable. Con estos resultados, se trabajaron los datos obtenidos en las curvas ROC generadas y se evaluaron los puntos de corte con su respectiva sensibilidad y especificidad para los parámetros clínicos indicados previamente (Tabla 3 ).

En la Tabla 4, se encuentran resumidos los OR (con su respectivo intervalo de $95 \%$ de confianza) donde además se expresa el coeficiente de regresión.

Finalmente, mediante análisis discriminante se encontró que la mejor combinación de los parámetros estudiados fue hematocrito, plaquetas y GOT. En la Tabla 5 se muestra la asociación entre la clasificación obtenida del análisis discriminante y la confirmación de infección por hantavirus. A partir de este análisis se obtuvo una sensibilidad de $90,9 \%$ (70,8\% - 98,8\%) y una especificidad de $81,6 \%(67,9 \%-91,2 \%)$.

\section{Discusión}

El aumento del hematocrito, la trombocitopenia, presencia de inmunoblastos observados en este estudio se condicen con los resultados obtenidos por Koster y cols. $(2001)^{5}$. Sin embargo, el aumento de GOT y prolongación del TTPa se podría considerar como un hallazgo en este estudio.

Los valores de sensibilidad y especificidad obtenidos para las plaquetas son comparables con la investigación efectuada por Navarrete y cols. ${ }^{2}$, que en su estudio obtuvieron $98 \%$ de sensibilidad con una especificidad de $74 \%$ para los valores de plaquetas en casos de sospecha por SCPH. Asimismo, se concluye que este parámetro puede ser utilizado como parámetro indicador de sospecha para el médico clínico. Por otra parte, al valor de punto de corte obtenido para los inmunoblastos, si bien no tiene una sensibilidad elevada (59\%), muestra una alta especificidad (89,8\%). Esta especificidad podría ser criterio de confirmación para casos negativos, ya que un valor menor a $15 \%$ de inmunoblastos sería indicativo de ausencia de infección por SCPH. Navarrete y cols. ${ }^{2}$, obtuvieron valores de sensibilidad de $25 \%$ y especificidad de $98 \%$ para inmunoblastos y en su trabajo fue el parámetro menos sensible.

A los puntos de cortes obtenidos, se les acompaña pares de sensibilidad/especificidad buenos para una prueba de cribado. Las plaquetas tienen la mejor sensibilidad en este estudio que se condice con la capacidad discriminatoria mencionada anteriormente. Cabe destacar que el punto de corte obtenido en este estudio $\left(<119 \times 103 / \mathrm{mm}^{3}\right)$ es menor al propuesto por la guía clínica de manejo de infección por hantavirus del Ministerio de Salud (MINSAL) de Chile $^{3}$. Dicha guía, no sugiere puntos de corte para los demás exámenes de laboratorio evaluados en este estudio.

El objetivo de la investigación era buscar valores pre- 
dictores que ayudasen al clínico en la pesquisa precoz de la enfermedad; en este sentido, la Tabla 4 muestra cómo cambia el riesgo de enfermedad por hantavirus por cada unidad de aumento en las variables estudiadas. Individuos con mayores valores de hematocrito, inmunoblastos y TTPA tendrían más riesgo de presentar enfermedad por hantavirus. Todas las variables anteriores presentan un OR de 1,1 lo que se traduce en que, por cada aumento en una unidad de la variable, el riesgo aumenta en $10 \%$.

En virtud de los resultados encontrados en esta investigación, se sugiere que exista una mayor disponibilidad de algunos de estos exámenes como el hemograma en centros de atención primaria o contar con un acceso a laboratorio expedito para estos casos.

\section{Conclusión}

Los cinco parámetros estudiados son buenos predictores de SCPH en pacientes con sospecha clínica de enfermedad por hantavirus y podrían ser útiles en hospitales de baja complejidad para rápido traslado a centros que cuenten con UPC. La combinación de hematocrito, plaquetas y GOT poseen sensibilidad y especificidad sobre $80 \%$. A la luz de los resultados obtenidos en este trabajo se sugiere realizar los exámenes estudiados ante una sospecha de enfermedad por hantavirus.

Agradecimientos: A Sergio Mella M, por su revisión de este manuscrito.

\section{Resumen}

Introducción: La infección por hantavirus es una zoonosis emergente, endémica en Chile, generando el síndrome cardiopulmonar por hantavirus ( $\mathrm{SCPH})$, caracterizado por disfunción cardiopulmonar con falla respiratoria rápidamente progresiva y altamente letal. Para una orientación clínica precoz del SCPH, debido a su poca especificidad en síntomas y ayudar al diagnóstico diferencial, se han estudiado algunos parámetros de laboratorio que puedan ser de utilidad. Objetivo: Identificar criterios del laboratorio como factores predictores del diagnóstico de SCPH en pacientes con sospecha de enfermedad por hantavirus. Metodología. Estudio de cohorte retrospectiva de 71 pacientes que ingresaron a Urgencia del Hospital Guillermo Grant Benavente. Se determinó la capacidad discriminativa de parámetros de laboratorio al momento de ingreso: recuento de plaquetas, hematocrito, inmunoblastos, TTPa y GOT. Resultados: Se encontraron diferencias significativas en los parámetros estudiados entre pacientes confirmados (n: 22) con respecto a los no confirmados (n: 49). Hematocrito, inmunoblastos, GOT y TTPa tuvieron un OR $>1$ y las plaquetas un $\mathrm{OR}<1$. La mejor combinación para predecir SCPH fue hematocrito, plaquetas y GOT con sensibilidad $90,9 \%$ y especificidad 81,6\%. Conclusión: Los cinco parámetros estudiados son buenos predictores de SCPH en pacientes con sospecha del mismo y podrían ser útiles en hospitales de baja complejidad para rápido traslado a centro que cuente con unidad de pacientes crítico.

\section{Referencias bibliográficas}

1.- Vaheri A, Strandin T, Hepojoki J, Sironen T, Henttonen H, Mäkelä S Et al. Uncovering the mysteries of hantavirus infections. Nat Rev Microbiol 2013; 11 (8): 539-50. PMID: 24020072.

2.- Navarrete M, Hott M, Caroca J, Leyton L. Correlación entre criterios clínicos y de laboratorio de casos notificados por sospecha de hantavirosis y resultado de la técnica de referencia. Rev Chilena Infectol 2016; 33 (33): 275-81. https://scielo.conicyt.cl/pdf/rci/v33n3/ art04.pdf.

3.- Ministerio de Salud. Guía Clínica de prevención, diagnóstico y tratamiento del SCPH (en línea). Resolución Exenta No 881.
Santiago, 12 de diciembre del 2013. Disponible en: http://www.minsal.cl/sites/default/files/files/ HANTA_imprimir.pdf.

4.- Departamento de Epidemiología. Minuta de situación epidemiológica de hantavirus, semanas 1-52. Ministerio de Salud de Chile; 2018.

5.- Koster F, Foucar K, Jelle B. Scott A, Chong Y $\mathrm{Y}$, Larson R, et al. Rapid presumptive diagnosis of hantavirus cardiopulmonary syndrome by peripheral blood smear., hematopathology/ diagnosis of hantavirus cardiopulmonary syndrome by peripherical blood smear review. Am J Clin Pathol 2001; 116: 665-72. doi:10.1309/CNWF-DC72-QYMR-M8DA.

6.- Gavrilovskaya I N, Gorbunova E E and Mackow E R. Pathogenic hantaviruses direct the adherence of quiescent platelets to infected endothelial cells. J Virol. 2010; 84 (9): 4832. doi: 10.1128/JVI.02405-09.

7.- Rasmuson J, Andersson C, Norrman E, Haney $\mathrm{M}$, et al. Time to revise the paradigm of hantavirus syndromes? Hantavirus pulmonary syndrome caused by European Hantavirus. Eur J Clin Microbiol Infect Dis. 2011; 30: 685-90. doi: 10.1007/s10096-010-1141-6.

8.- Kaya S, Yagei D, Uyar, I Y1lmaz H, Engin $\mathrm{A}$, Öngürü $\mathrm{P}$ et al. Can hantavirus infections be predicted on admission to hospital? J Med Virol.2012; 84:1790-6. doi: 10.1002/jmv.23390.

9.- Hosmer, W. Lemeshow, S. Assesing the fit of the model. En: Hosmer W. Lemeshow S. Applied Logistic Regression. $2^{\circ}$ Edición. Wiley-Interscience Publication; 2000. P 162. 